\title{
Bone Marrow Stromal Antigen 2 is a Potential Unfavorable Prognostic Factor for High-Grade Glioma
}

This article was published in the following Dove Press journal: OncoTargets and Therapy

\author{
Haiping Shi \\ Ke Luo* \\ Wei Huang
}

Department of Neurosurgery, Suining Central Hospital, Suining, Sichuan, People's Republic of China

*These authors contributed equally to this work
Correspondence: Haiping Shi

Suining Central Hospital, \#I27 Desheng

West Road, Suining, Sichuan 629000,

People's Republic of China

Tel +86-I5244933180

Email haipingshihps@I26.com
Background: Bone marrow stromal antigen 2 (BST2) is considered as a transmembrane glycoprotein and plays essential roles in innate immunity. It has been recently reported that up-regulation of BST2 was associated with the development of breast carcinoma. However, the clinical significance of BST2 in glioma has not been identified. The purpose of the present study is to explore the expression pattern and the role of BST2 in the progression of high-grade glioma.

Methods: Expression levels of BST2 were tested in glioma tissues by analyzing the GEO database and immunohistochemistry staining. The prognostic role of BST2 in glioma was evaluated through univariate and multivariate analyses. In vitro and in vivo assays were conducted to confirm the role of BST2 on promoting glioma proliferation.

Results: The mRNA level of BST2 was higher in glioma tissues than that in nontumorous brain tissues. High protein level of BST2 was correlated with larger tumor size and advanced WHO grade. Glioma patients with a high BST2 level had worse overall survival. In addition, BST2 was defined as an independent risk factor for glioma prognosis. Cellular and xenograft studies revealed that BST2 can significantly promote glioma proliferation.

Conclusion: Our study revealed that a high BST2 expression level was closely related to the unfavorable clinical features and poor prognosis of high-grade glioma patients. BST2 may serve as an invaluable prognostic indicator and novel therapeutic target for glioma treatment considering its membrane localization.

Keywords: high-grade glioma, BST2, proliferation, prognosis

\section{Introduction}

Glioma is one of the most common primary malignancies in the central nervous system of adults, which is characterized with a poor prognosis and high recurrence incidence. Overall survival of glioma differs significantly according to its WHO grade. For the low-grade (WHO grade II) glioma, the median survival time is 10-17 years, ${ }^{1,2}$ with a 10 -year overall survival rate at $47 \%{ }^{3}$ However, for high-grade gliomas (WHO grade III-IV), median overall survival is only 15-36 months due to its rapid proliferation and invasion. ${ }^{4,5}$ Furthermore, although the prognosis seems optimistic for low-grade glioma patients, most of them will develop into high-grade ones within 5-10 years. ${ }^{6}$ However, current treatment for high-grade glioma is far from satisfactory, as we described before. ${ }^{7,8}$ Therefore, figuring out novel prognosis indicators and elucidating their signaling pathways are invaluable for understanding its progression mechanisms as well as developing novel therapeutic targets. 
Bone marrow stromal cell antigen 2 (BST2) is a kind of cell surface glycoprotein. ${ }^{9}$ It contains a cytoplasmic $\mathrm{N}$-terminal region, a transmembrane domain, a coiled-coil extracellular region, and a C-terminal glycosylphosphatidylinositol anchor domain. ${ }^{10}$ BST2 was originally reported to serve as a specific surface marker of differentiated B cells, ${ }^{11}$ which is rarely to be detected in other parenchymatous tissues such as the brain. The major role of BST2 is functioning as a component to defend against invading pathogens such as virus. ${ }^{12}$ However, aberrant expression of BST2 was indeed detected in several pathological tissues. For example, high BST2 expression in the digestive system is correlated with malignancies and poor prognosis. ${ }^{13}$ Similarly, BST2 was demonstrated to promote growth and metastasis of breast cancer. ${ }^{14}$ Of note, a recent study reported that BST2 can remarkably enhance cisplatin resistance of nasopharyngeal cancer, perhaps through modulating the NF-kB signaling pathway, indicating its potential as a novel drug target. ${ }^{15}$ However, the clinical significance of BST2 in glioma has not been clarified yet.

Here we investigated the expression pattern of BST2 in clinical glioma tissues from both mRNA and protein levels. The GEO database demonstrated that the BST2 transcription level is remarkably upregulated in gliomas compared with nontumorous brain tissues. Univariate and multivariate analyses revealed BST2 as an independent risk factor of glioma prognosis. Finally, cellular assays implicated a significant effect of BST2 on enhancing glioma cell proliferation, which is further confirmed by xenograft experiments.

\section{Patients and Methods}

\section{Patients and Samples}

This retrospective study was approved by the Ethic Committee of Suining Central Hospital in 2018. Written informed consent was obtained from all patients or their direct relatives. Formalin fixed paraffin-embedded glioma tissues were collected from those who underwent surgical resection during 2007-2017 $(\mathrm{n}=85)$. All the enrolled patients satisfied the following criteria: i) did not receive neoadjuvant therapy before surgery; ii) pathologically diagnosed as high-grade glioma; iii) survived for at least 6 months since the date of surgery to exclude surgeryrelated death; and iv) follow-up information was intact.

\section{Antibodies and Materials}

The BST2 antibody was purchased from Invitrogen (Pittsburgh, PA, USA) and diluted at 1:200 for immunohistochemistry staining. Anti-beta-actin antibody was purchased from Santa Cruz and used at 1:10,000 dilution for Western blotting assay. The siRNA sequences used in this study are listed as follows:

Scramble-siRNA: UUCUCCGAACGUGUCACGU, BST2-siRNA\#1: CCUGCAACCACACUGUGAU, BST2-siRNA\#2: GCUCCUGAUCAUCGUGAUU,

The sequence of BST2-shRNA was CCGGTGCTCCT GATCATCGTGATTCCTCGAGGAATC-ACGATGATCA GGAGCATTTTTG, which was packaged into lentivirus by Genomeditech (Shanghai, China).

\section{Immunohistochemistry (IHC) and IHC Analysis}

To detect BST2 protein expression in sample tissues, IHC examination was conducted as described previously. ${ }^{16}$ Briefly, deparaffinized and rehydrated slides were treated with $10 \mathrm{mM}$ citrate buffer $(\mathrm{pH} 6.0)$ at $95^{\circ} \mathrm{C}$ for antigen recovery, followed by quenching the endogenous peroxidase activity with $3 \% \mathrm{H}_{2} \mathrm{O}_{2}$ incubation. $5 \%$ Fetal bovine serum (FBS) was then used to block the nonspecific binding sites. Slides were then incubated with primary antibodies at $4^{\circ} \mathrm{C}$ overnight and a DAB staining kit was finally used to detect the immunoreactivity.

The IHC evaluation was performed independently by two pathologists based on both the staining intensity and positive percentage. Briefly, the staining intensity was scored as 1 (weak staining), 2 (moderate staining), or 3 (strong staining). The percentage of positive stained cells was scored as 1 ( $0-25 \%$ positive), 2 ( $26-50 \%$ positive), 3 (50-75\% positive), and 4 (76-100\% positive). The expression level of BST2 was finally evaluated by multiplying the two scores above (ranging from 1-12).

\section{Cell Culture and Transfection}

We obtained five cell lines in this study, including normal human astroglia (NHA) cells and four human glioma cell lines (T98G, U87, U251, and A172). All cell lines were purchased from the Cell Bank of the Chinese Academy of Sciences (Shanghai, China). Cells were cultured in DMEM supplemented with $10 \%$ fetal bovine serum (Gibco, Gaithersburg, MD, USA) and 1\% penicillin/streptomycin under $5 \% \mathrm{CO}_{2}$ in a 37-degree incubator.

For the transfection assay, we enrolled a mock group (only added the transfection reagent Lipofectamine 2000), a vector group (transfected with pcDNA3.1 vector plasmids), and a scramble group (transfected with scramble 
siRNA) as control groups, respectively. All transfections were achieved by using Lipofectamine 2000 reagent (Invitrogen, Pittsburgh, PA, USA). ${ }^{17}$ Transiently transfected cells were further subjected to MTT assays or Edu assays to evaluate cell viabilities. Additionally, cells were transfected with specific shRNA lentivirus to obtain stable cell lines with BST2-knockdown for further xenograft experiments.

\section{Cell Proliferation Assay}

The proliferation capacities of U87 and U251 cells were assessed using both MTT (3-(4,5-dimethylthiazol-2-yl)2,5-diphenyl tetrazolium bromide) (Tiangen, China) assay and Edu assay. Each cell line was divided into two groups in overexpression tests, one transfected with pcDNA3.1-vector, and the other one transfected with pcDNA3.1-BST2 plasmids. Similarly, for knockdown assay, each cell line was divided into three parallel groups, including the scramble-siRNA group, BST2-siRNA\#1 group, and BST2-siRNA\#2 group.

Approximately 2000 transfected cells were seeded into a 96-well plate in $100 \mu \mathrm{L}$ DMEM and MTT measurements were performed 8, 24, 48, 72, and 96 hours after seeding according to the manufacturer's protocol. ${ }^{18}$ The absorbance was measured at $490 \mathrm{~nm}$ using a microplate reader. Each experiment was conducted in triplicate.

As for the Edu assay, transfected cells were seeded into another 96-well plate and cultured in complete DMEM for 24 hours before adding the Edu reagent. Cells were then incubated, fixed, permeabilized, washed, and supplemented with a reaction cocktail according to the manufacturer's instructions from the Edu assay kit (RiboBio, Guangzhou, China). ${ }^{19}$ DAPI reagent was used to visualize the nuclei. Finally, images of cells were recorded under a microscope and the merged percentage of cells were counted. Each experiment was conducted in triplicate for three independent times.

\section{Western Blot}

Total protein in cultured cells was extracted with NP-40 lysis buffer containing protease inhibitors and phosphatase inhibitors. Approximately $20 \mu \mathrm{g}$ of total protein was subjected to Western blot by separating them in 12\% SDS-PAGE and electro-transferred onto polyvinylidene difluoride membranes (Millipore, Billerica, MA, USA). Membranes were blocked with 5\% FBS for 1 hour and then incubated with the primary antibodies overnight. Then the membrane was incubated with corresponding secondary antibodies and the enhanced chemiluminescence (ECL) was added to detect specific bands using films. ${ }^{20}$

\section{Xenograft Model of Glioma in Nude Mice}

Male BALB/c nude mice (6 weeks old) were purchased from the Shanghai Institute of Materia Medical, Chinese Academy of Sciences, and housed under specific pathogen-free conditions. The experimental protocol was approved by the Suining Central Hospital Animal Center. Mice were randomly assigned to the experimental group and the control group, and $5 \times 10^{6}$ stable-transfected cells were subcutaneously implanted into mice. ${ }^{21}$ Tumor volumes were measured every 5 days to obtain the growth curve. After 20 days, all mice were sacrificed, and all xenografts were isolated and weighed.

\section{Statistical Analysis}

Statistical analysis was performed with SPSS 20.0 (SPSS Inc., Chicago, IL, USA) or Graphpad Prism software 6.0. Overall survival time was expressed as mean $\pm \mathrm{SD}$ (standard deviation) and median (interquartile range). Patients were divided into different subgroups according to cut-off values. For example, the cut-off age was set by median age (51 years), and the BST2 expression cut-off value was determined according to ROC (receiver operating characteristic) curve generated by IHC staining. The correlations between BST2 expression and patients' clinicopathological characteristics were tested by using Chi-square test. Univariate survival analysis was performed using the Kaplan-Meier method, and the significance of the difference between the groups was analyzed using the Log rank test. The independent prognostic significance of each variable was assessed using Cox proportional hazards regression model. Data from cellular and xenograft experiments were compared by Student's $t$-test. $P<0.05$ was regarded as statistically significant and indicated by an asterisk in the tables and figures.

\section{Results}

\section{Patients' Characteristics}

Among the 85 enrolled high-grade glioma patients, there were 30 females (35.3\%) and 55 males (64.7\%) with the age ranging from 24-69 years old at the time of diagnosis. There were 58 cases $(68.2 \%)$ with the largest tumor diameter less than $5.0 \mathrm{~cm}$, while the other 27 cases $(31.8 \%)$ had a tumor diameter larger than $5.0 \mathrm{~cm}$. Among all the patients, 38 cases $(44.7 \%)$ were classified as WHO grade III, and the other 47 cases $(55.3 \%)$ with WHO grade IV. The subgroup analyses of patients with different WHO grades are provided in 
Supplementary Table S1. We also assessed the disease severity by calculating Karnofsky Performance Score (KPS), ${ }^{22}$ which indicated that 50 patients $(58.8 \%)$ had a KPS score less than 90 , and 35 cases $(41.2 \%)$ a KPS score of 90 or above. The surgery pattern of our cohort was also retrieved, showing that 37 patients $(43.5 \%)$ underwent gross total resection, 31 cases $(36.5 \%)$ underwent subtotal resection, while the other 17 cases (20.0\%) underwent partial resection or biopsy. As for adjuvant therapy after surgical treatment, 14 cases (16.5\%) underwent radiotherapy, 19 cases (22.4\%) underwent chemotherapy, and the other 52 cases (61.2\%) underwent combined radio- and chemotherapy. The follow-up time ranged from 6-62 months.

A

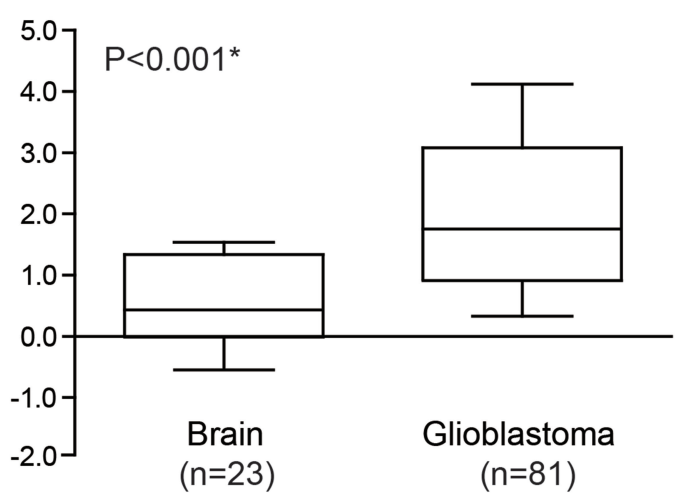

C

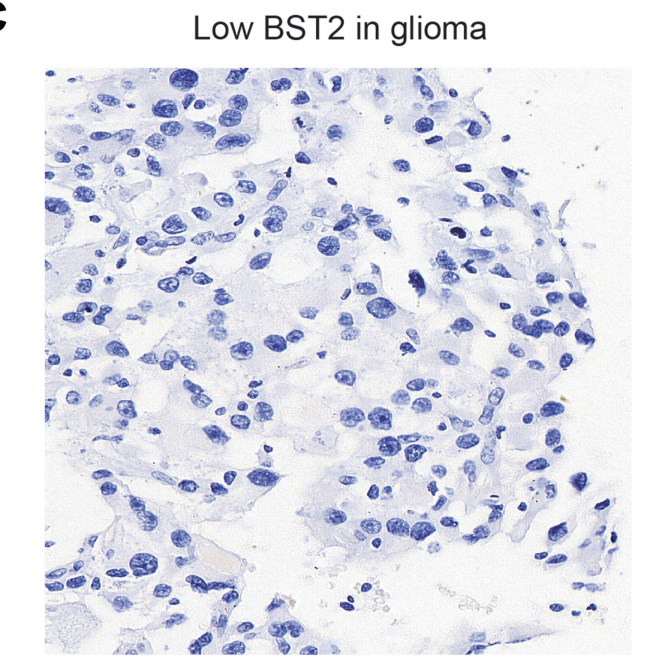

BST2 Expression is Upregulated in

\section{Gliomas and Correlated with Tumor Size}

By analyzing the GEO database (GDS1962), ${ }^{23}$ we found that transcription of the BST2 gene in glioblastoma was significantly higher than that in normal brains (Figure 1A). Therefore, we tested the protein expression of BST2 in the FFPE glioma tissues by IHC method and plotted an ROC curve to distinguish high- and low-BST2 patients (Figure 1B). IHC results showed predominant localization of BST2 protein in cytoplasm and membrane (Figure $1 \mathrm{C}$ and D). According to the ROC curve, we classified cases into a low-BST2 group (IHC score $<5$ ) and a high-BST2 group (IHC score $\geq 5$ ). Accordingly, 45 patients were sub-grouped as low BST2 expression and the other 40 patients as high BST2 expression.

B

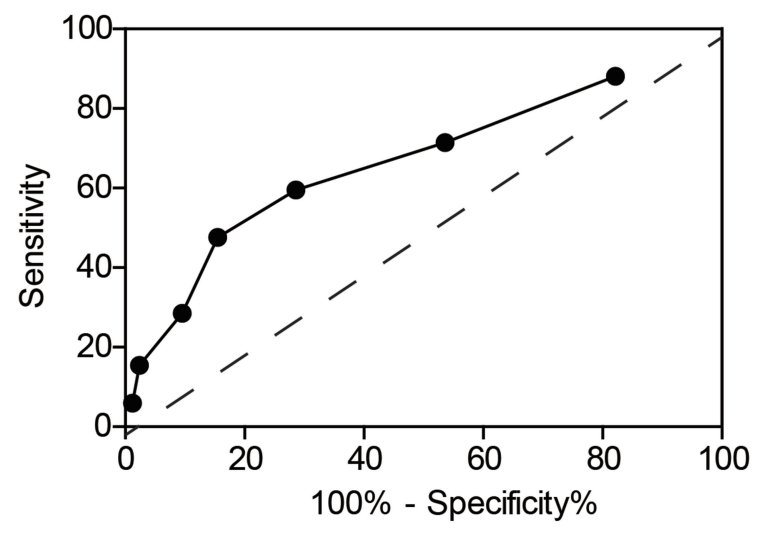

D

High BST2 in glioma

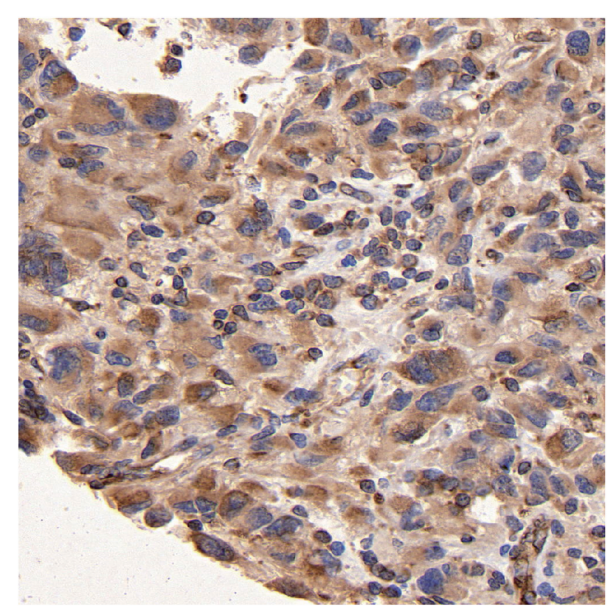

Figure I BST2 is upregulated in glioma tissues. (A) GEO analysis demonstrated that BST2-mRNA is higher in glioblastomas compared with normal brain tissues. (B) ROC curve was plotted to identify the cut-off value to divide patients into high-BST2 group and low-BST2 group based on the IHC scores. The area under curve is 0.67 and IHC score $=5.0$ was identified as the cut-off score. (C and D) Representative IHC images of BST2 staining in gliomas and brain tissues. Magnification: $400 X$. *Indicates P<0.05 by Student's $t$-test. 
We next evaluated the correlations between BST2 expression and clinicopathological features of all the patients (Table 1). A significant correlation between tumor size and BST2 was observed, showing that patients with higher BST2 are more likely to possess larger tumor size $(P=0.045)$. Similarly, higher BST2 is positively correlated with advanced WHO grade $(P=0.033)$. In contrast, BST2 showed no significant correlation with patients' age, gender, Karnofsky score, or surgical method. Of note, although the subgroup analyses did not provide any statistically significant correlation between tumor size and BST2 expression (Supplementary Table S1), which may be caused by limited case numbers, patients with a lower BST2 level did exhibit a trend of smaller tumor size in either WHO grade III or grade IV subgroups.

Table I Correlations Between Clinical Characteristics and BST2 Expression in Glioma Patients

\begin{tabular}{|c|c|c|c|c|}
\hline \multirow[t]{2}{*}{ Variables } & \multirow{2}{*}{$\begin{array}{l}\text { Patients } \\
(n=85)\end{array}$} & \multicolumn{2}{|c|}{ BST2 Expression } & \multirow[t]{2}{*}{$P$ value } \\
\hline & & $\begin{array}{l}\text { Low } \\
(n=45)\end{array}$ & $\begin{array}{l}\text { High } \\
(n=40)\end{array}$ & \\
\hline Gender & & & & 0.688 \\
\hline Female & 30 & 15 & 15 (50.0\%) & \\
\hline Male & 55 & 30 & 25 (45.5\%) & \\
\hline Age (years) & & & & 0.740 \\
\hline$\leq 50$ & 42 & 23 & $19(45.2 \%)$ & \\
\hline$>50$ & 43 & 22 & 21 (48.8\%) & \\
\hline Tumor size & & & & $0.045^{*}$ \\
\hline$\leq 5.0 \mathrm{~cm}$ & 58 & 35 & $23(39.7 \%)$ & \\
\hline$>5.0 \mathrm{~cm}$ & 27 & 10 & $17(63.0 \%)$ & \\
\hline WHO grade & & & & $0.033^{*}$ \\
\hline III & 38 & 25 & $13(34.2 \%)$ & \\
\hline IV & 47 & 20 & 27 (57.4\%) & \\
\hline Karnofsky score & & & & 0.516 \\
\hline$<90$ & 50 & 25 & $25(50.0 \%)$ & \\
\hline$\geq 90$ & 35 & 20 & $15(42.9 \%)$ & \\
\hline Surgery & & & & 0.233 \\
\hline GTR & 37 & 19 & 18 (48.6\%) & \\
\hline STR & 31 & 14 & $17(54.8 \%)$ & \\
\hline PR/biopsy & 17 & 12 & $5(29.4 \%)$ & \\
\hline Adjuvant therapy & & & & 0.116 \\
\hline Radiotherapy & 14 & 10 & $4(28.6 \%)$ & \\
\hline Chemotherapy & 19 & 12 & 7 (36.8\%) & \\
\hline Combined therapy & 52 & 23 & $29(55.8 \%)$ & \\
\hline
\end{tabular}

Note: *Statistically significant by Chi-square test.

Abbreviations: BST2, Bone marrow stromal antigen 2; GTR, gross total resection; STR, subtotal resection; PR, partial resection.

\section{Higher BST2 Indicates Unfavorable Overall Survival of Glioma Patients}

To determine the clinical relevance of BST2 expression in glioma, overall survival data was next assessed using Kaplan-Meier method (Table 2). Seventy-three patients (85.9\%) died by the end of follow-up. The median overall survival time of our enrolled cohort was 22 months, and the 3 -year overall survival rate was $33.6 \%$. Of note, survival analysis suggested that patients with high BST2 expression in glioma tissue had shorter overall survival time than those with low expression (Figure 2A, $P=0.014$ ). The 3-year overall survival rate was $44.3 \%$ for low-BST2 patients, while it was $21.9 \%$ for high-BST2 patients. Additionally, high BST2 expression showed an unfavorable effect on

Table 2 Univariate Analysis for Overall Survival of Glioma Patients

\begin{tabular}{|c|c|c|c|c|}
\hline \multirow[t]{2}{*}{ Variables } & \multirow{2}{*}{$\begin{array}{l}\text { Patients } \\
(n=85)\end{array}$} & \multicolumn{2}{|c|}{$\begin{array}{l}\text { Overall Survival Time } \\
\text { (Months) }\end{array}$} & \multirow[t]{2}{*}{$P$ value } \\
\hline & & Mean \pm s.d & Median & \\
\hline Gender & & & & 0.197 \\
\hline Female & 30 & $33.8 \pm 3.4$ & 36.0 & \\
\hline Male & 55 & $25.8 \pm 2.6$ & 19.0 & \\
\hline Age (years) & & & & 0.169 \\
\hline$\leq 50$ & 42 & $25.3 \pm 2.9$ & 17.0 & \\
\hline$>50$ & 43 & $31.9 \pm 2.8$ & 34.0 & \\
\hline Tumor size & & & & $0.005^{*}$ \\
\hline$\leq 5.0 \mathrm{~cm}$ & 58 & $32.5 \pm 2.6$ & 34.0 & \\
\hline$>5.0 \mathrm{~cm}$ & 27 & $21.1 \pm 3.1$ & 15.0 & \\
\hline WHO grade & & & & $0.002 *$ \\
\hline III & 38 & $36.2 \pm 3.2$ & 39.0 & \\
\hline IV & 47 & $22.9 \pm 2.4$ & 15.0 & \\
\hline Karnofsky score & & & & 0.618 \\
\hline$<90$ & 50 & $29.6 \pm 2.8$ & 22.0 & \\
\hline$\geq 90$ & 35 & $27.9 \pm 3.1$ & 22.0 & \\
\hline Surgery & & & & $0.032^{*}$ \\
\hline GTR & 37 & $33.6 \pm 3.4$ & 35.0 & \\
\hline STR & 31 & $25.9 \pm 2.7$ & 22.0 & \\
\hline PR/biopsy & 17 & $22.5 \pm 4.5$ & 13.0 & \\
\hline Adjuvant therapy & & & & $0.012^{*}$ \\
\hline Radiotherapy & 14 & $27.3 \pm 5.7$ & 19.0 & \\
\hline Chemotherapy & 19 & $17.8 \pm 3.2$ & 12.0 & \\
\hline Combined therapy & 52 & $33.0 \pm 2.5$ & 34.0 & \\
\hline BST2 expression & & & & $0.014 *$ \\
\hline Low & 45 & $32.7 \pm 3.2$ & 34.0 & \\
\hline High & 40 & $24.5 \pm 2.4$ & 22.0 & \\
\hline
\end{tabular}

Note: *Statistically significant by Log rank test.

Abbreviations: GTR, gross total resection; STR, subtotal resection; PR, partial resection; BST2, Bone marrow stromal antigen 2 . 
A Overall survival (BST2 expression)

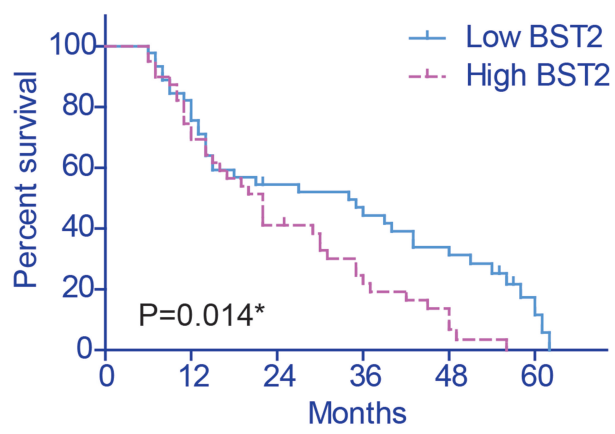

C Overall survival (Age)

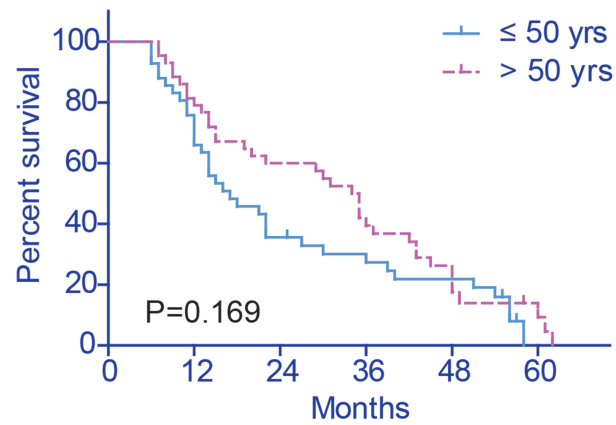

E Overall survival (WHO grade)

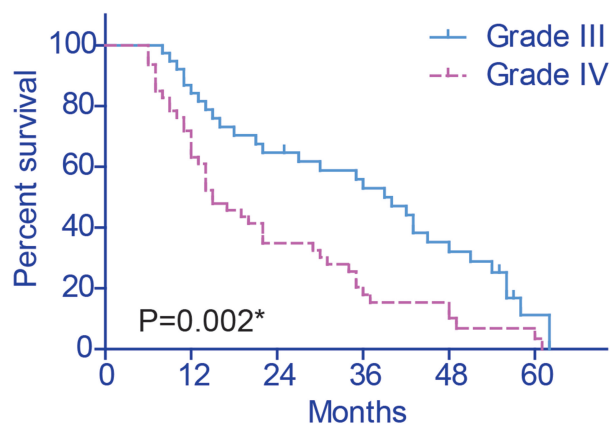

G Overall survival (Surgery)

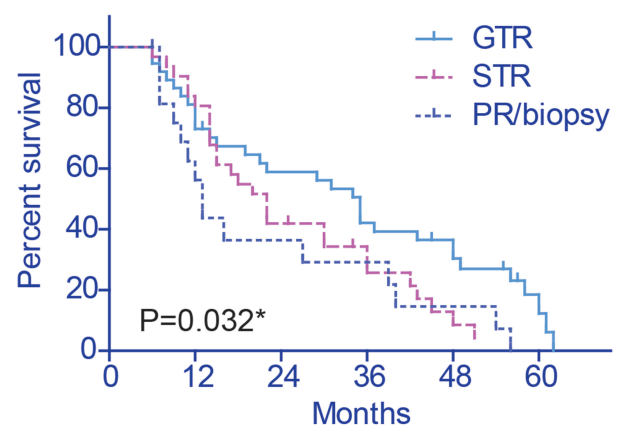

B Overall survival (Sex)

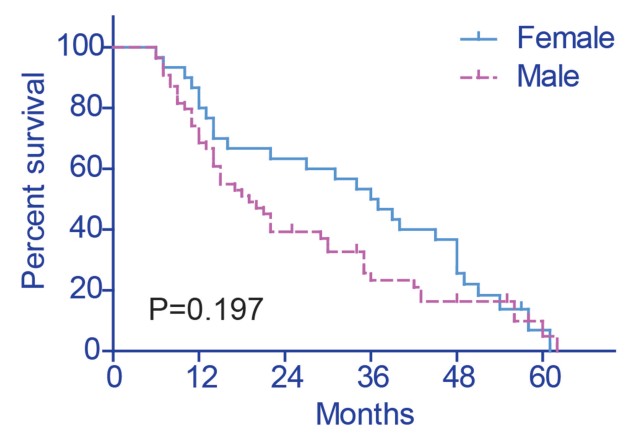

D Overall survival (Tumor size)

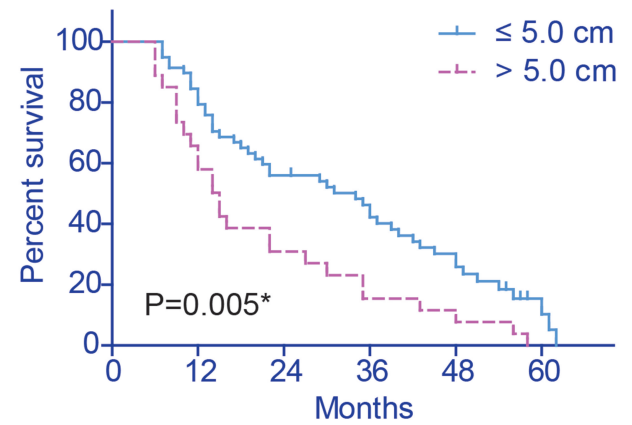

F Overall survival (Karnofsky score)

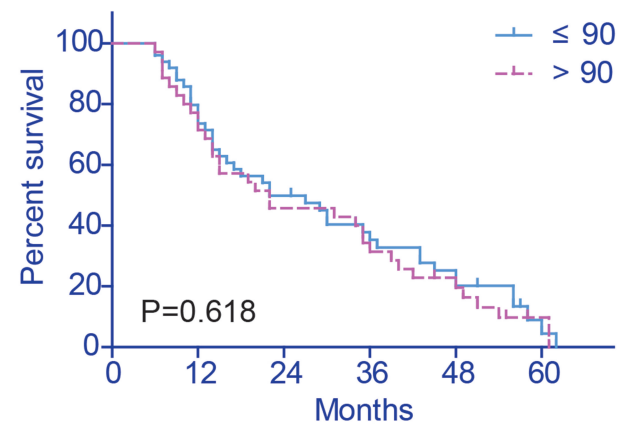

H Overall survival (Adjuvant therapy)

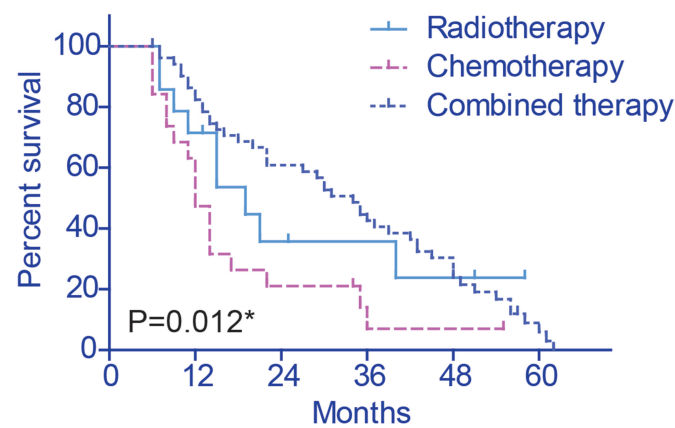

Figure 2 Overall survival of glioma patients. The survival curves of our entire cohort were plotted by Kaplan-Meier method and prognostic effect of each variables was assessed by Log rank test, including BST2 level (A), patients' sex (B), age (C), tumor size (D), WHO grade (E), KPS score (F), resection pattern (G) and adjuvant therapy (H), respectively. *Indicates $\mathrm{P}<0.05$ by Log rank test. 
patients' survival in subgroup analyses compared to those with low BST2 expression, especially for WHO grade III patients (mean survival time $28.60 \pm 5.16$ vs $40.20 \pm 3.81$ months, $P=0.070$, Supplementary Table S2).

The prognostic effects of other related variables were also analyzed (Figure $2 \mathrm{~B}-\mathrm{H})$, which indicated significant effects of tumor size $(P=0.005)$, WHO grade $(P=0.002)$, surgery pattern $(P=0.032)$, and adjuvant therapy $(P=0.012)$ on patients' survival. Briefly, patients with larger tumor size, advanced WHO grade, who underwent palliative resection, or who were treated with insufficient postoperative therapy showed poorer clinical outcomes (Table 2). In contrast, no statistically significant effect was observed regarding patients' sex, age, or Karnofsky score.

Multivariate analysis was further conducted to identify independent prognostic factors by subjecting those significant variables of Kaplan-Meier analysis into a Cox regression model (Table 3). Accordingly, a high BST2 level was confirmed as an independent risk factor $(\mathrm{HR}=1.859,95 \%$ $\mathrm{CI}=1.031-3.352, P=0.039)$. Besides, a larger tumor size $(\mathrm{HR}=1.744,95 \% \mathrm{CI}=1.041-2.923, P=0.035)$, advanced WHO grade $\quad(\mathrm{HR}=2.255, \quad 95 \% \quad \mathrm{CI}=1.300-3.912$, $P=0.004)$, and insufficient surgical resection $(\mathrm{HR}=1.799$, 95\% CI=1.302-2.486, $P<0.001)$ were also independently associated with poorer prognosis. In contrast, combined postoperative therapy exhibited significant benefit for patients' overall survival compared to monotherapy $(\mathrm{HR}=0.411,95 \% \mathrm{CI}=0.245-0.689, P=0.001)$.

\section{BST2 Enhances Glioma Cell Proliferation in vitro and in vivo}

Considering that BST2 expression is associated with patient survival in human high-grade glioma, we sought to further understand the potential role of BST2 glioma cell lines. Immunoblotting data firstly revealed significantly higher

Table 3 Multivariate Analysis for the Prognostic Factors of Glioma Patients

\begin{tabular}{|c|c|c|c|}
\hline Variables & HR & $95 \% \mathrm{Cl}$ & $P$ value \\
\hline Tumor size $(>5.0 \mathrm{~cm}$ vs $\leq 5.0 \mathrm{~cm})$ & 1.744 & $1.04 I-2.923$ & $0.035 *$ \\
\hline WHO grade (Grade IV vs grade III) & 2.255 & $1.300-3.912$ & $0.004 *$ \\
\hline Surgery (GTR/STR vs PR/biopsy) & 1.799 & $1.302-2.486$ & $<0.001 *$ \\
\hline $\begin{array}{l}\text { Therapy (Combined vs radio-/ } \\
\text { chemotherapy) }\end{array}$ & 0.411 & $0.245-0.689$ & $0.001 *$ \\
\hline BST2 expression (High vs low) & 1.859 & $1.031-3.352$ & $0.039 *$ \\
\hline
\end{tabular}

Note: *Statistically significant by Cox regression test.

Abbreviations: $\mathrm{HR}$, hazard ratio; $\mathrm{Cl}$, confidence intervals; $\mathrm{BST} 2$, Bone marrow stromal antigen 2 .
BST2 protein levels in glioma cell lines compared with nontumorous NHA cells (Figure 3A). Among them, U87 and U251 showed the highest endogenous BST2 level, thus we selected these two cell lines for further experiments. We next tested whether transfecting pcDNA3.1 vector or scramble-siRNA had any effect on glioma cells. As expected, pcDNA3.1 vector or scramble-siRNA transfected cells showed comparable expression levels of BST2 with non-transfected cells (Figure 3B). Therefore, we chose vector-transfected cells as the control group for further overexpression assays, while scramble-siRNA transfected cells were chosen as the control group for knockdown assays.

After verifying the transfection efficacies of plasmid constructs in U87 (Figure 3C) and U251 (Figure 3D) cells, we conducted MTT experiments and found that overexpressing BST2 significantly enhanced the proliferation capacities of both cell lines (Figure $3 \mathrm{E}$ and $\mathrm{F}$ ). Consistently, Edu assays demonstrated an elevated cell viability after transfecting BST2 plasmids, indicating that BST2 promotes glioma cell proliferation (Figure 3G).

Additionally, we conducted BST2 knockdown by using siRNAs in U87 (Figure 4A) and U251 cells (Figure 4B), respectively. Then MTT assay was performed to evaluate the potential effect of silencing BST2 on the two cell lines (Figure 4C and D), which showed that knockdown of BST2 attenuated cell proliferation capacity compared with the control group. Similarly, Edu results further confirmed that silencing BST2 impaired cell division process (Figure 4E). We then established the stable cell line of U87 and U251 cells by using BST2-shRNA lentivirus and conducted xenograft experiments by subcutaneous injection into nude mice. Tumor growth curves were plotted and showed an impaired grow rate in the BST2-shRNA group (Figure 4F and G). Consistently, 20 days after subcutaneous injection, isolated tumor volume in the BST2shRNA group was significantly smaller than that control group.

\section{Discussion}

Rapid proliferation and invasion lead to an unsatisfactory outcome of high-grade glioma patients. Identifying molecules associated with glioma progression will be invaluable for risk evaluation and individual therapy development. BST2 had been initially reported to act as an effective nuclear factor kappa binding (NF-кB) activator and participates in DNA transcription. ${ }^{24,25}$ Besides, BST2 participates in cell-cell interactions due to its role on regulating the adhesion of monocytes to endothelial 
A

NHA T98G U87 U251 A172

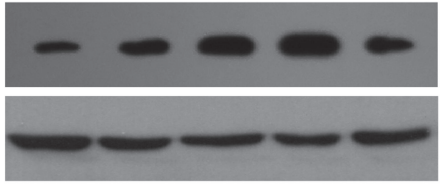

Anti-BST2

Anti- $\beta$-actin

C

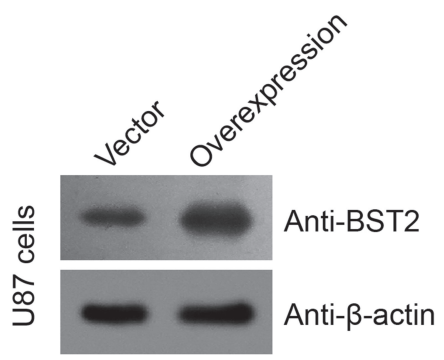

E

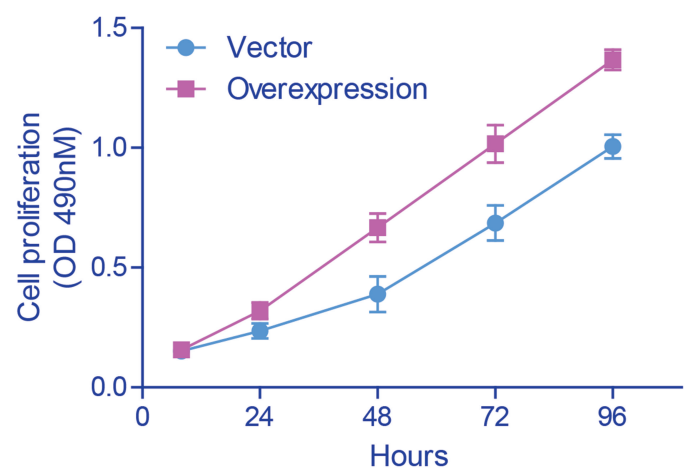

B

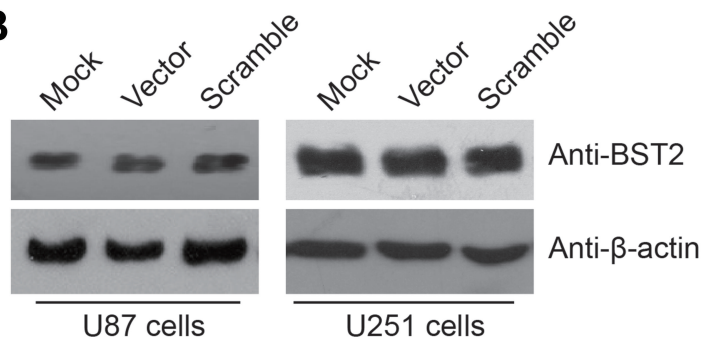

D

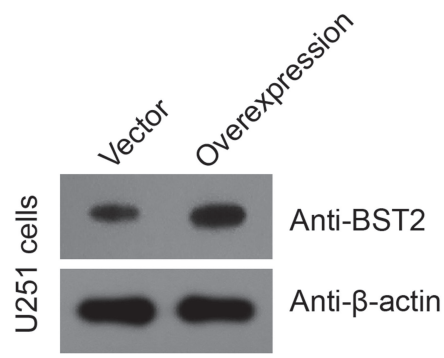

$\mathbf{F}$ MTT assay (U251 cells)

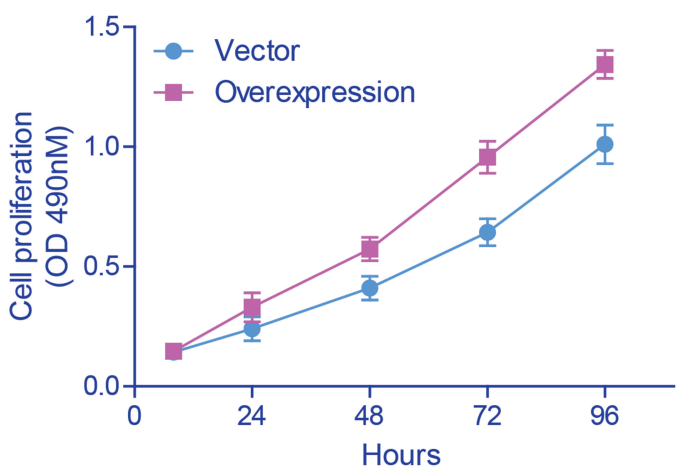

Vector Overexpression

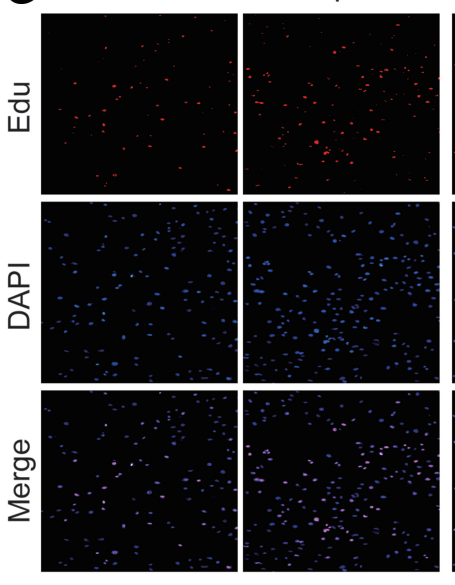

U87 cells

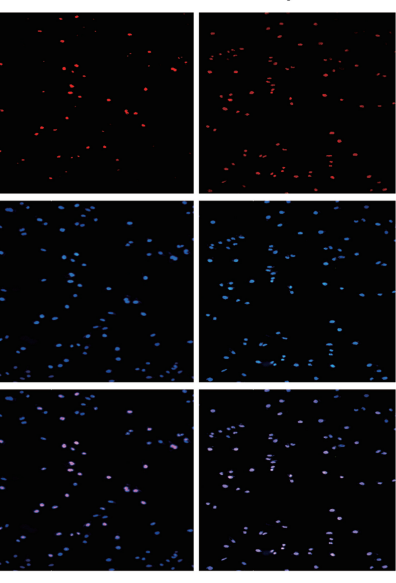

U251 cells

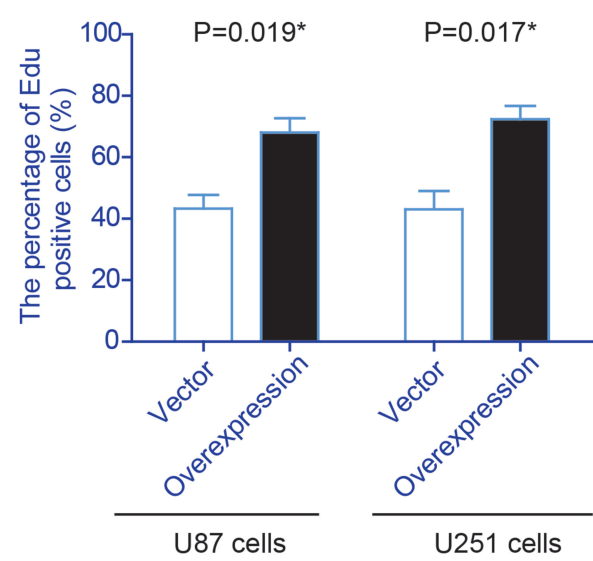

Figure 3 Overexpressing BST2 promotes glioma cell proliferation.(A) Immunoblotting data exhibited the protein levels of BST2 in different cells, indicating a lower BST2 level in normal human astroglia (NHA) cells than that in four glioma cell lines. (B) U87 and U25I cells were transfected with pcDNA3.I-vector plasmids or scramble-siRNA, both showed comparable BST2 expression levels to the cells treated with transfection reagent (mock group). (C and D) Transfection efficiencies of pcDNA3.I-BST2 plasmids in U87 and U25I cells were verified using pcDNA3.I-vector as control. (E and F) MTT assays were conducted to compare proliferation capacities of BST2-overexpressing cells and vectoroverexpressing cells, which showed that transfecting BST2 significantly enhanced cell viability in both U87 and U25I cells. (G) Edu experiments were performed and demonstrated consistent results with MTT data, on that overexpressing BST2 can upregulate cell proliferation capacity. *Indicates P<0.05 by Student's $t$-test. 
A

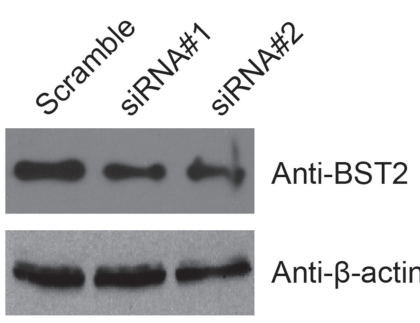

C

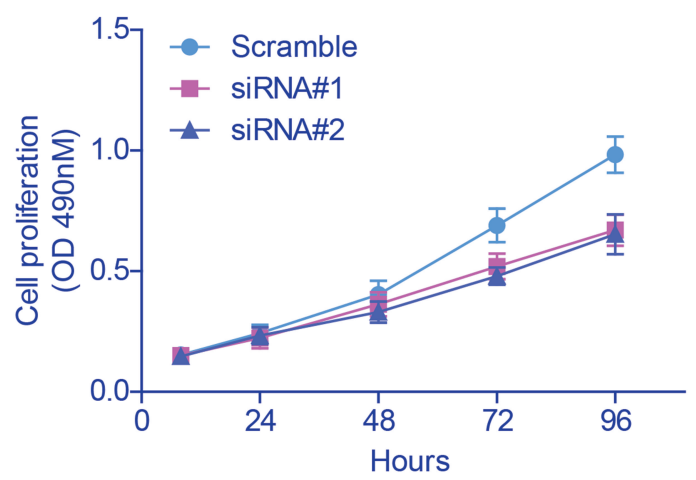

B

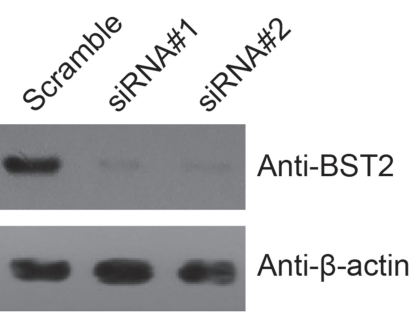

D

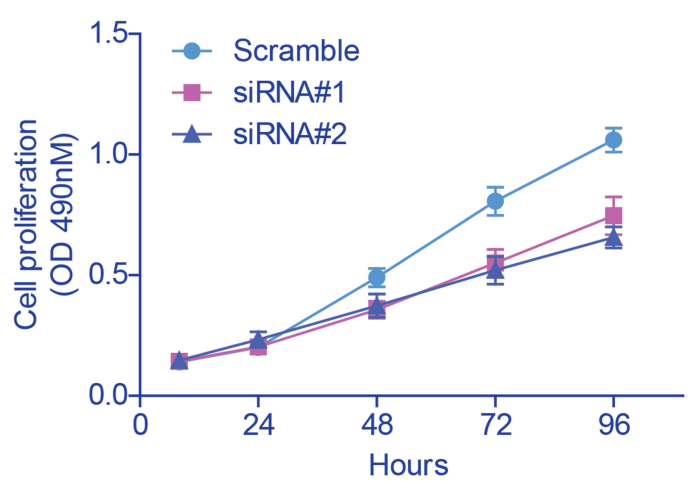

\section{E} Scramble siRNA\#1 siRNA\#2 Scramble siRNA\#1
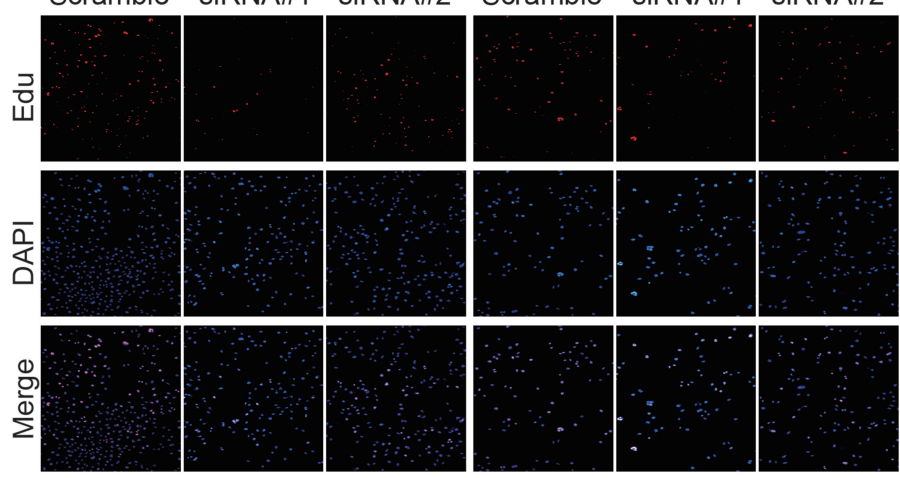

U87 cells

U251 cells

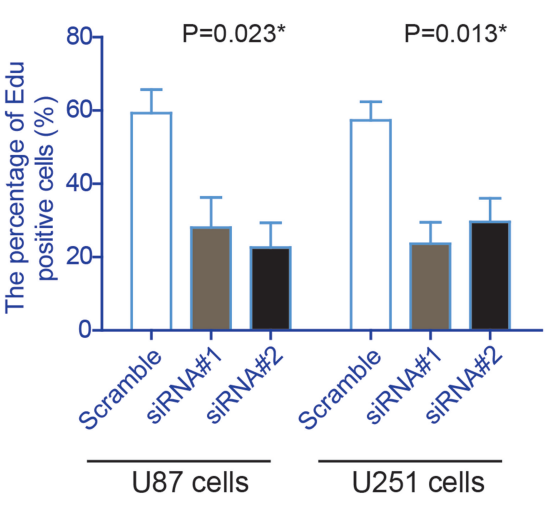

$\mathbf{F}$

Xenograft growth curve (U87)
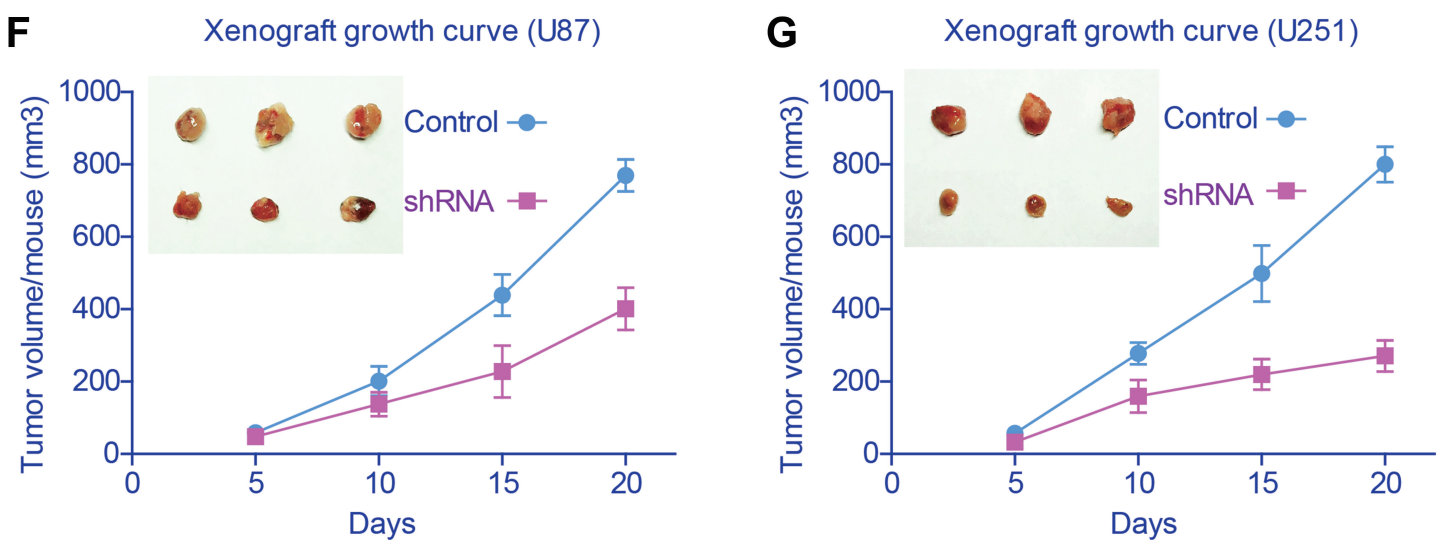

Figure 4 Silencing BST2 attenuates glioma cell proliferation both in vitro and in vivo.(A and B) Human glioma U87 and U25I cells were transfected with BST2-specific siRNAs using scramble siRNA as control, and their transfection efficiencies were tested by Western Blot. (C and D) The proliferation curves of transfected U87 and U25I cells were plotted based on MTT data, which indicated that silencing BST2 inhibited cell proliferation. (E) Edu experiments showed that glioma cells transfected with BST2siRNAs had impaired cell proliferation capacities compared to those transfected with scramble siRNAs. (F and $\mathbf{G})$ The growth curve of implanted xenografts was plotted, which showed that BST2-shRNA transfected U87 cells possessed an attenuated growth rate. Similarly, U25I cells with BST2-shRNA transfection also showed impaired growth capacity compared with control cells. *Indicates $\mathrm{P}<0.05$ by One-way ANOVA test or Student's $t$-test. 
cells. ${ }^{26,27}$ Both NF-KB activation and cell-cell adhesion are important regulators of tumor progression.

Here we retrieved an elevated transcription level of BST2 according to GEO datasets, and demonstrated that BST2 protein was predominantly expressed in plasma membrane of high-grade glioma tissues. Consistent with our data, Wainwright et $\mathrm{al}^{28}$ previously reported that BST2 expression is upregulated on mRNA level in high grade glioma compared with that in low grade human astrocytoma. Furthermore, we initially analyzed the relationship between BST2 expression level and clinicopathological characteristics of glioma patients. It revealed that higher expression BST2 was correlated with larger tumor size and advanced WHO grade for high-grade glioma patients. Additionally, clinical data demonstrated that BST2 expression might serve as an important prognosis predictor for high-grade glioma patients.

Taking into consideration that high-grade glioma are characterized by high proliferative capability, elevated BST2 may therefore be involved in tumor growth. We next verified its pro-oncogenic effect by silencing BST2 in U87 and U251 human glioma cells. As expected, silencing BST2 led to a significantly attenuated proliferation capacity of both cell lines. The effect of BST2 on glioma growth was further confirmed by using a xenograft model. There have been several possible functional mechanisms on BST2's role in promoting tumor growth. First, BST2 can facilitate cancer cell adhesion to adjacent cells and the extracellular matrix, thus promoting formation of primary tumors. ${ }^{29}$ Second, BST2 may promote tumor proliferation and attenuate cell apoptosis by activating NF- $\mathrm{\kappa B}$ signaling pathways. $^{15,30}$ Third, BST2 may also potentiate tumor growth by modulating secretion of certain soluble signaling molecules such as IFN- $\alpha$ and TNF- $\alpha$, which affects the tumor microenvironment. ${ }^{31}$ Additionally, a recent study reported the effect of BST2 on positively regulating cyclin D1 and ERK/MAPK signaling pathways, and both can enhance tumor cell proliferation. ${ }^{32}$ Therefore, we believe that BST2 can simultaneously promotes tumor growth through multiple molecular pathways.

Besides glioma, it has been previously reported that BST2 is highly expressed and exerts oncogenic functions in several other malignancies including breast cancer, lung cancer, and colorectal cancer. ${ }^{33}$ For example, BST2 promotes progression of thyroid cancer through direct p21 repression. ${ }^{34}$ Besides proliferation, BST2 was found to enhance the invasion capacity of gastric cancer cells. ${ }^{30,35}$ Moreover, inhibition of BST2 could suppress tumorigenesis in tumor-bearing mice models, emphasizing its role as a potential drug target. ${ }^{36,37}$ Taken together, an abnormally high protein level of BST2 is closely associated with the progress of certain malignancies, and more intensive studies focused on its fundamental mechanisms will be important for future clinical applications.

Our study has several limitations. First, all cases were enrolled from a single medical center which may lead to regional bias. Second, here we focused on investigating BST2's clinical role and did not dig into its underlying molecular mechanisms on modulating glioma growth. More studies will be necessary to further illuminating its functional mechanisms to provide experimental evidence for inhibitor development.

\section{Conclusion}

In conclusion, our results established a positive correlation between BST2 level and tumor grades in glioma patients, and verified that BST2 is a novel predictor for their overall survival. In vitro and in vivo assays revealed that BST2 can enhance the proliferation of glioma.

\section{Data Sharing Statement}

Data will be available upon request.

\section{Statement of Ethics}

This research was conducted ethically in accordance with the World Medical Association Declaration of Helsinki. Subjects have given their written informed consent and the study protocol regarding human specimen and cell lines were approved by the Ethic Committee of Suining Central Hospital. Mice experimental protocol was conducted under the supervision of Suining Central Hospital Animal Center following the GB/T 35,892-2018 guideline (Laboratory animal-Guideline for ethical review of animal welfare).

\section{Funding}

There is no funding to report.

\section{Disclosure}

The authors declare no conflicts of interest.

\section{References}

1. Ohgaki H, Kleihues P. Population-based studies on incidence, survival rates, and genetic alterations in astrocytic and oligodendroglial gliomas. J Neuropathol Exp Neurol. 2005;64(6):479-489. doi:10. 1093/jnen/64.6.479

2. Duffau H. Diffuse Low-Grade Gliomas in Adults. Springer; 2017. 
3. Smoll NR, Gautschi OP, Schatlo B, Schaller K, Weber DC. Relative survival of patients with supratentorial low-grade gliomas. NeuroOncology. 2012;14(8):1062-1069. doi:10.1093/neuonc/nos144

4. Bleeker FE, Molenaar RJ, Leenstra S. Recent advances in the molecular understanding of glioblastoma. $J$ Neurooncol. 2012;108 (1):11-27. doi:10.1007/s11060-011-0793-0

5. Kline C, Felton E, Allen IE, Tahir P, Mueller S. Survival outcomes in pediatric recurrent high-grade glioma: results of a 20 -year systematic review and meta-analysis. $J$ Neurooncol. 2018;137(1):103-110. doi:10.1007/s11060-017-2701-8

6. Maher EA, Furnari FB, Bachoo RM, et al. Malignant glioma: genetics and biology of a grave matter. Genes Dev. 2001;15(11):1311-1333. doi:10.1101/gad.891601

7. Shi H, Zhang S. Expression and prognostic role of orphan receptor GPR110 in glioma. Biochem Biophys Res Commun. 2017;491 (2):349-354. doi:10.1016/j.bbrc.2017.07.097

8. Duan J, Huang W, Shi H. Positive expression of KIF20A indicates poor prognosis of glioma patients. Onco Targets Ther. 2016;9:6741-6749. doi:10.2147/ott.s115974

9. Dietrich I, Hosie MJ, Willett BJ. The role of BST2/tetherin in feline retrovirus infection. Vet Immunol Immunopathol. 2011;143 (34):255-264. doi:10.1016/j.vetimm.2011.06.020

10. Hiramatsu K, Serada S, Kobiyama K, et al. CpG oligodeoxynucleotides potentiate the antitumor activity of anti-BST2 antibody. Cancer Sci. 2015;106(10):1474-1478. doi:10.1111/cas.12738

11. Holmgren AM, Miller KD, Cavanaugh SE, Rall GF. Bst2/tetherin is induced in neurons by type $\mathrm{i}$ interferon and viral infection but is dispensable for protection against neurotropic viral challenge. $J$ Virol. 2015;89(21):11011-11018. doi:10.1128/JVI.01745-15

12. Lewinski MK, Jafari M, Zhang H, Opella SJ, Guatelli J. Membrane anchoring by a c-terminal tryptophan enables HIV-1 vpu to displace bone marrow stromal antigen 2 (BST2) from sites of viral assembly. J Biol Chem. 2015;290(17):10919-10933. doi:10.1074/jbc.M114. 630095

13. Mukai S, Oue N, Oshima T, et al. Overexpression of transmembrane protein BST2 is associated with poor survival of patients with esophageal, gastric, or colorectal cancer. Ann Surg Oncol. 2017;24 (2):594-602. doi:10.1245/s10434-016-5100-Z

14. Mahauad-Fernandez WD, DeMali KA, Olivier AK, Okeoma CM. Bone marrow stromal antigen 2 expressed in cancer cells promotes mammary tumor growth and metastasis. Breast Cancer Res. 2014;16 (6):493.

15. Kuang C-M, Fu X, Hua Y-J, et al. BST2 confers cisplatin resistance via NF- $\kappa$ B signaling in nasopharyngeal cancer. Cell Death Dis. 2017;8(6):e2874e. doi:10.1038/cddis.2017.271

16. Liu Z, Sun R, Zhang X, et al. Transcription factor 7 promotes the progression of perihilar cholangiocarcinoma by inducing the transcription of c-Myc and FOS-like antigen 1. E Bio Medicine. 2019;45:181-191. doi:10.1016/j.ebiom.2019.06.023

17. Liu H, Liu Z, Li K, et al. TBL1XR1 predicts isolated tumor cells and micrometastasis in patients with TNM stage I/II colorectal cancer. $J$ Gastroenterol Hepatol. 2017;32(9):1570-1580. doi:10.1111/ jgh.13749

18. Liu H, Xu Y, Zhang Q, et al. Correlations between TBL1XR1 and recurrence of colorectal cancer. Sci Rep. 2017;7:44275. doi:10.1038/ srep44275

19. Zhang Q, Fan H, Zou Q, et al. TEAD 4 exerts pro-metastatic effects and is negatively regulated by miR6839-3p in lung adenocarcinoma progression. J Cell Mol Med. 2018;22(7):3560-3571. doi:10.1111/ jcmm.13634

20. Sun R, Liu Z, Qiu B, et al. Annexin10 promotes extrahepatic cholangiocarcinoma metastasis by facilitating EMT via PLA2G4A/ PGE2/STAT3 pathway. E Bio Medicine. 2019;47:142-155. doi:10.1016/j.ebiom.2019.08.062
21. Zhang Q, Fan H, Liu H, et al. WNT5B exerts oncogenic effects and is negatively regulated by miR-5587-3p in lung adenocarcinoma progression. Oncogene. 2019;1-14.

22. Karnofsky DA, Abelmann WH, Craver LF, Burchenal JH. The use of the nitrogen mustards in the palliative treatment of carcinoma. With particular reference to bronchogenic carcinoma. Cancer. 1948;1 (4):634-656. doi:10.1002/1097-0142(194811)1:4<634::AIDCNCR2820010410>3.0.CO;2-L

23. Sun L, Hui AM, Su Q, et al. Neuronal and glioma-derived stem cell factor induces angiogenesis within the brain. Cancer Cell. 2006;9 (4):287-300. doi:10.1016/j.ccr.2006.03.003

24. Fernandez-Oliva A, Finzi A, Haim H, Menendez-Arias L, Sodroski J, Pacheco B. HIV-1 adapts to replicate in cells expressing common marmoset APOBEC3G and BST2. J Virol. 2016;90(2):725-740. doi:10.1128/JVI.02431-15

25. Sayeed A, Luciani-Torres G, Meng Z, Bennington JL, Moore DH, Dairkee SH. Aberrant regulation of the BST2 (Tetherin) promoter enhances cell proliferation and apoptosis evasion in high grade breast cancer cells. PLoS One. 2013;8(6):e67191. doi:10.1371/journal. pone.0067191

26. Hammonds J, Wang JJ, Yi H, Spearman P. Immunoelectron microscopic evidence for Tetherin/BST2 as the physical bridge between HIV-1 virions and the plasma membrane. PLoS Pathog. 2010;6(2): e1000749. doi:10.1371/journal.ppat.1000749

27. Tokarev A, Suarez M, Kwan W, Fitzpatrick K, Singh R, Guatelli J. Stimulation of NF-kappaB activity by the HIV restriction factor BST2. J Virol. 2013;87(4):2046-2057. doi:10.1128/JVI.02272-12

28. Wainwright DA, Balyasnikova IV, Han Y, Lesniak MS. The expression of BST2 in human and experimental mouse brain tumors. Exp Mol Pathol. 2011;91(1):440-446. doi:10.1016/j.yexmp.2011.04.012

29. Mahauad-Fernandez WD, DeMali KA, Olivier AK, Okeoma CM. Bone marrow stromal antigen 2 expressed in cancer cells promotes mammary tumor growth and metastasis. Breast Cancer Res. 2014;16 (6):1-18. doi:10.1186/s13058-014-0493-8

30. Liu W, Cao Y, Guan Y, Zheng C. BST2 promotes cell proliferation, migration and induces NF- $\mathrm{BB}$ activation in gastric cancer. Biotechnol Lett. 2018;40(7):1015-1027. doi:10.1007/s10529-018-2562-z

31. Demoulin S, Herfs M, Delvenne P, Hubert P. Tumor microenvironment converts plasmacytoid dendritic cells into immunosuppressive/ tolerogenic cells: insight into the molecular mechanisms. J Leukoc Biol. 2013;93(3):343-352. doi:10.1189/jlb.0812397

32. Xu X, Wang Y, Xue F, et al. BST2 promotes tumor growth via multiple pathways in hepatocellular carcinoma. Cancer Invest. 2020;(just-accepted):1-21.

33. Cai D, Cao J, Li Z, et al. Up-regulation of bone marrow stromal protein 2 (BST2) in breast cancer with bone metastasis. BMC Cancer. 2009;9:102. doi:10.1186/1471-2407-9-102

34. Herbert C, Am S, Mm B, et al. Anti-inflammatory and anti-remodelling effects of ISU201, a modified form of the extracellular domain of human BST2, in experimental models of asthma: association with inhibition of histone acetylation. PLoS One. 2014;9 (3):e90436. doi:10.1371/journal.pone.0090436

35. Loschko J, Schlitzer A, Dudziak D, et al. Antigen delivery to plasmacytoid dendritic cells via BST2 induces protective T cell-mediated immunity. J Immunol. 2011;186(12):6718-6725. doi:10.4049/ jimmunol.1004029

36. Lo Sicco C, Reverberi D, Villa F, et al. Circulating healing $(\mathrm{CH})$ cells expressing BST2 are functionally activated by the injury-regulated systemic factor HGFA. Stem Cell Res Ther. 2018;9(1):300. doi:10.1186/s13287-018-1056-1

37. Mansouri M, Viswanathan K, Douglas JL, et al. Molecular mechanism of BST2/tetherin downregulation by K5/MIR2 of Kaposi's sarcoma-associated herpesvirus. J Virol. 2009;83(19):9672-9681. doi:10.1128/JVI.00597-09 


\section{Publish your work in this journal}

OncoTargets and Therapy is an international, peer-reviewed, open access journal focusing on the pathological basis of all cancers, potential targets for therapy and treatment protocols employed to improve the management of cancer patients. The journal also focuses on the impact of management programs and new therapeutic agents and protocols on patient perspectives such as quality of life, adherence and satisfaction. The manuscript management system is completely online and includes a very quick and fair peer-review system, which is all easy to use. Visit http://www.dovepress.com/ testimonials.php to read real quotes from published authors.

Submit your manuscript here: https://www.dovepress.com/oncotargets-and-therapy-journal 